\title{
A Brief Review on Primordial Black Holes as Dark Matter
}

\author{
Pablo Villanueva-Domingo *, Olga Mena and Sergio Palomares-Ruiz \\ Instituto de Física Corpuscular (IFIC), CSIC-Universitat de València, Paterna, Spain
}

Primordial black holes (PBHs) represent a natural candidate for one of the components of the dark matter (DM) in the Universe. In this review, we shall discuss the basics of their formation, abundance and signatures. Some of their characteristic signals are examined, such as the emission of particles due to Hawking evaporation and the accretion of the surrounding matter, effects which could leave an impact in the evolution of the Universe and the formation of structures. The most relevant probes capable of constraining their masses and population are discussed.

Keywords: primordial black holes, dark matter, cosmology, accretion, $21 \mathrm{~cm}$ cosmology, gravitational waves, cosmic microwave background, microlensing

OPEN ACCESS

Edited by: Fabrizio Nesti,

University of L'Aquila, Italy

Reviewed by:

Maxim Yurievich Khlopov, Southern Federal University, Russia Vyacheslav Ivanovich Dokuchaev, Institute for Nuclear Research (RAS),

Russia

*Correspondence: Pablo Villanueva-Domingo pablo.villanueva.domingo@ gmail.com

Specialty section:

This article was submitted to Cosmology,

a section of the journal Frontiers in Astronomy and Space Sciences

Received: 15 March 2021 Accepted: 11 May 2021

Published: 28 May 2021

Citation:

Villanueva-Domingo $P$, Mena $O$ and

Palomares-Ruiz S (2021) A Brief Review on Primordial Black Holes as

Dark Matter.

Front. Astron. Space Sci. 8:681084. doi: 10.3389/fspas.2021.681084

\section{INTRODUCTION}

The hypothesis of the formation of black holes (BHs) in the early Universe was first suggested in 1967 by Zeldovich and Novikov (Zel'dovich and Novikov, 1967), and independently by Hawking in 1971 (Hawking, 1971). Soon after, the possibility that primordial black holes (PBHs) could account for at least part of the DM became obvious (Chapline, 1975; Meszaros, 1975). At that time, the DM question started to be outlined as one of the fundamental problems in cosmology [see, e.g., the reviews in Sanders (2010); Bertone and Hooper (2018)]. DM (partly) composed by PBHs constitutes an exciting possibility, presenting an enormous number of observable signatures which can constrain its parameter space, as shall be detailed in Sec. $\mathbf{6}$. The variety of phenomenological effects produced by PBHs allows placing stringent bounds on the abundance of PBHs, usually indicated by the energy fraction of DM as PBHs, $f_{\mathrm{PBH}}=\Omega_{\mathrm{PBH}} / \Omega_{\mathrm{DM}}$, with $\Omega_{\mathrm{PBH}}$ and $\Omega_{\mathrm{DM}}$ the ratios of energy densities and critical density. Moreover, since PBHs are usually expected to be formed before nucleosynthesis, BBN constraints on the baryon abundance do not apply to them, as they do not intervene in the nucleosynthesis of elements, and thus, can be regarded as non-baryonic DM (Carr and Kühnel, 2020). Several recent reviews are devoted to discuss PBHs in great detail (see, e.g., Sasaki et al., 2018; Carr and Kühnel, 2020; Carr et al., 2020; Green and Kavanagh, 2021).

Shortly after the first detection of gravitational waves from a merger of $\sim 30 M_{\odot}$ BHs by LIGO (Abbott et al., 2016), the question whether these could be of primordial nature was raised (Bird et al., 2016). Analysis of posterior data from the gravitational wave detectors LIGO and Virgo showed that the detected mergers are compatible with the hypothesis of their components being of primordial nature, although there is no strong preference over stellar BHs (Sasaki et al., 2016; Ali-Haïmoud et al., 2017; Clesse and García-Bellido, 2017; Clesse and García-Bellido, 2018; Garcia-Bellido et al., 2020; De Luca et al., 2020b; De Luca et al., 2021; Wong et al., 2021). PBHs with a lognormal mass function have been claimed to better fit data than BHs from astrophysical origin (Dolgov et al., 2020), although this is in contrast to the results of Hall et al. (2020), and a mixed population seems compatible or even favored (Hütsi et al., 2021).

Unlike stellar BHs, formed from the collapse of a massive star, which can present masses only above $\sim 3 M_{\odot}$ [the Tolman-Oppenheimer-Volkoff limit (Oppenheimer and Volkoff, 1939; Tolman, 
1939)], PBHs could be produced with any mass. Thus, a positive measurement of a $\mathrm{BH}$ with a mass lower than $\sim 3 M_{\odot}$ would be a confirmation of the existence of primordial, non-stellar $\mathrm{BHs}$ (Clesse and García-Bellido, 2018). PBHs could also conform intermediate mass $\mathrm{BHs}$, with masses between $\sim 10^{2} M_{\odot}$ and $10^{5} M_{\odot}$, too massive to be originated from a single star. It is the case of the merger event of BHs with masses $\sim 60 M_{\odot}$ and $\sim 80 M_{\odot}$, producing a remnant $\mathrm{BH}$ of $\sim 150 M_{\odot}$, in a so far mostly unobserved range of masses (Abbott et al., 2020). Furthermore, PBHs could constitute the seeds for super massive black holes (SMBHs), present in the nuclei of most galaxies, with masses ranging from $10^{5} M_{\odot}$ to $10^{10} M_{\odot}$, and already existing at redshifts $z>6$ (Carr and Silk, 2018). Such massive objects can hardly be produced from accreting stellar remnant BHs (Volonteri, 2010). However, the existence of massive enough $\mathrm{PBH}$ may act as seeds for the $\mathrm{SMBHs}$, from which they could have grown by accretion.

$\mathrm{PBH}$ formation is already present in standard cosmologies, although extremely unlikely. However, their production usually requires some exotic inflationary scenarios or physics beyond the Standard Model (BSM) in order to obtain a large enough abundance. The typically considered formation mechanism of $\mathrm{PBH}$ arises from the direct collapse of primordial fluctuations, whose power is enhanced at small scales as a consequence of some inflationary potential, as we shall further comment on below. There are, however, other scenarios which naturally predict a population of $\mathrm{PBH}$ as a result of phase transitions in the early Universe and by the collapse of topological defects (Hawking et al., 1982; Hawking, 1989; Polnarev and Zembowicz, 1991; Garriga et al., 2016; Deng et al., 2017; Deng and Vilenkin, 2017). Hence, the existence of $\mathrm{PBH}$ would provide valuable hints about the still unknown physics of the very early Universe (see, e.g., Polnarev and Khlopov, 1985; Khlopov, 2010; Belotsky et al., 2014), and may allow to probe high-energy scales and supersymmetric theories (Ketov and Khlopov, 2019).

In this review, the most relevant aspects of PBHs as DM are briefly discussed, such as the mechanism of formation in Section 2, the initial abundance and mass distribution in Section 3, the process of accretion in Section 4 and other $\mathrm{PBH}$ features, which could leave imprints on different observables, in Section 5. Current observational constraints on their population are summarized in Section 6, and we conclude in Section 7.

\section{FORMATION AND CONDITIONS OF COLLAPSE}

The mass of a $\mathrm{BH}$ which collapsed in the early Universe depends on its formation time. A BH can be characterized by an extremely dense amount of matter in a very compact region, i.e., within the wellknown Schwarzschild radius, $R_{S}=2 G M_{\mathrm{PBH}} / c^{2} \sim 3 M_{\mathrm{PBH}} / M_{\odot} \mathrm{km}$. Thus, the mean density inside that region can be estimated as $\rho_{S}=M_{\mathrm{PBH}} /\left(4 \pi R_{S}^{3} / 3\right) \sim 10^{18}\left(M / M_{\odot}\right)^{-2} \mathrm{~g} \mathrm{~cm}^{-3}$. On the other hand, the mean density of the universe in the radiation era scales with time as $\rho_{c} \sim 10^{6}(t / \mathrm{s})^{-2} \mathrm{~g} \mathrm{~cm}^{-3}$. In order to have $\mathrm{PBH}$ formation, densities at least of the order of the mean inside the BH horizon, $\rho_{c} \sim \rho_{S}$, are needed. Therefore, the mass of the resulting
PBHs should be of the order of the horizon mass at that time, i.e., the mass within a region of the size of the Hubble horizon, $M_{\mathrm{PBH}} \sim M_{\mathrm{H}}$ (Carr, 1975), which is defined as

$$
M_{\mathrm{H}}=\frac{4}{3} \bar{\rho}\left(\frac{c}{H}\right)^{3}=\frac{c^{3}}{2 G H} \sim 10^{15} \mathrm{~g}\left(\frac{t}{10^{-23} \mathrm{~s}}\right) .
$$

PBHs with masses of $\sim M_{\odot} \simeq 2 \times 10^{33} \mathrm{~g}$ would have been formed at around the QCD phase transition, at $t \sim 10^{-6} \mathrm{~s}$. Since the PBH mass is roughly given by the mass within the horizon, it means that fluctuations entering the horizon can collapse into PBHs. A detailed calculation shows that $M_{\mathrm{PBH}}=\gamma M_{\mathrm{H}}$, where the proportionality factor $\gamma$ depends on the details of gravitational collapse, and gets values lower than 1 . Early estimates showed that it can be approximated as $\gamma \simeq c_{s}^{3 / 2} \simeq 0.2$, with $c_{s}=1 / 3$ the sound speed at the radiation epoch (Carr, 1975) More refined results show that the PBH mass is given by a scaling relation with the overdensity $\delta$, $M_{\mathrm{PBH}}=\kappa\left(\delta-\delta_{c}\right)^{\alpha}$ (Niemeyer and Jedamzik, 1998; Niemeyer and Jedamzik, 1999), where $\kappa$ and $\alpha$ are order unity constants, which depend on the background cosmology and on the shape of the perturbation (Niemeyer and Jedamzik, 1998; Niemeyer and Jedamzik, 1999; Musco et al., 2005), and $\delta_{c} \simeq c_{s}^{2}$ is the collapse threshold [see, e.g., Escrivà et al., 2021; Musco et al., 2021 for recent accurate computations]. Figure 1 depicts a sketch of the process of $\mathrm{PBH}$ formation.

Since PBHs are formed when fluctuations cross the horizon by the time of formation, $t_{f}$, their mass can be related to the wavelength of perturbations. When the mode of wavenumber $k$ crosses the horizon, the condition $a\left(t_{f}\right) H\left(t_{f}\right)=k$ holds. Since the mass of $\mathrm{PBHs}$ is proportional to the horizon mass at the moment of formation, $M_{\mathrm{PBH}} \propto \gamma H^{-1}$, at the radiation dominated era (Sasaki et al., 2018),

$$
M_{\mathrm{PBH}} \simeq 30 M_{\odot}\left(\frac{\gamma}{0.2}\right)\left(\frac{2.9 \times 10^{5} \mathrm{Mpc}}{k}\right)^{2} .
$$

Hence, probing a given scale $k$ could constrain a $\mathrm{PBH}$ population of its corresponding mass. Furthermore, an enhancement in the power spectrum around that scale would result in a large number of PBHs of such masses. In this review, we shall only consider PBHs formed during the radiation era. Those produced before inflation ends would have been diluted due to their negligible density during the inflationary accelerated expansion. PBHs formed during the matter-dominated era, or in an early matter domination era previous to the radiation era, have also been considered in the literature, and may have different imprints, since the conditions of collapse are less restrictive, and could start from smaller inhomogeneities (see, e.g., Green and Kavanagh, 2021).

\section{ABUNDANCE AND MASS FUNCTION OF PBHS}

It is possible to estimate the initial abundance of $\mathrm{PBH}$ at the moment of formation, taking into account all overdensities above the threshold for collapse, $\delta_{c} \simeq 1 / 3$. Assuming a Gaussian probability distribution, $P(\delta)$, for the overdensities with 


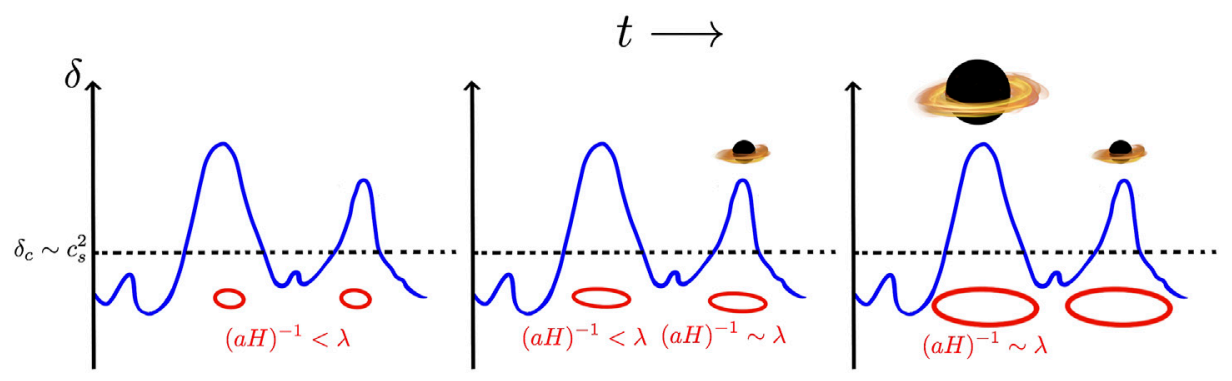

FIGURE 1 | Sketch of the formation of PBHs from overdensities for three different successive moments. When fluctuations larger than a critical threshold $\delta_{c} \sim c_{s}^{2}$ enter the horizon, i.e., their wavelength $\lambda=2 \pi / k$ (which characterizes the size of the perturbation) is of the order of the Hubble horizon (aH) ${ }^{-1}$, the overdense region collapses and a PBH is produced. As can be seen in Eqs 1, 2, longer modes (large $\lambda$, low k) enter the horizon later and lead to more massive PBHs.

variance $\sigma^{2}(M)$ at a mass scale $M$, the initial abundance, defined as $\beta\left(M_{\mathrm{H}}\right)=\rho_{\mathrm{PBH}}\left(t_{f}\right) / \rho_{\mathrm{tot}}\left(t_{f}\right)$, is given by (Sasaki et al., 2018; Green and Kavanagh, 2021)

$\beta\left(M_{\mathrm{PBH}}\right) \simeq \gamma \int_{\delta_{c}}^{\infty} P(\delta) d \delta \simeq \gamma \sqrt{\frac{2}{\pi}} \frac{\sigma\left(M_{\mathrm{PBH}}\right)}{\delta_{c}} \exp \left[-\frac{\delta_{c}^{2}}{2 \sigma^{2}\left(M_{\mathrm{PBH}}\right)}\right]$,

where in the last equality, $\sigma\left(M_{\mathrm{H}}\right) \ll \delta_{c}$ has been assumed. For the standard cosmological scenario with an initial scale invariant power spectrum, at CMB scales, the amplitude of the fluctuations is around $\sigma\left(M_{\mathrm{PBH}}\right) \sim 10^{-5}$, leading to $\beta \sim 10^{-5} \exp \left(-10^{10}\right)$, which is completely negligible (Green, 2014). Therefore, in order to have a relevant population of $\mathrm{PBHs}$, larger values of the initial power spectrum are needed. On the other hand, the assumption of a Gaussian distribution may not be consistent with enhanced fluctuations and the presence of PBHs, so deviations are unavoidable (Franciolini et al., 2018; De Luca et al., 2019b) [except for specific inflation models presenting an inflection point Atal and Germani (2019)]. Non-gaussianities could have a great impact on the initial fraction and lead to a larger population, as well as leaving detectable signatures in gravitational waves (Cai et al., 2019). Finally, note that the above formula follows the simple Press-Schechter approach, whereas to account for the non-universal nature of the threshold, the use of peak theory provides more accurate results (Green et al., 2004; Germani and Musco, 2019). Its validity, however, is limited to relatively narrow power spectra, while broader spectra require the use of non-linear statistics (Germani and Sheth, 2020).

Nonetheless, although the initial fraction $\beta$ is a very small quantity, since matter and radiation densities scale differently with redshift $\left[\propto(1+z)^{3}\right.$ and $\propto(1+z)^{4}$ respectively], the $\mathrm{PBH}$ contribution can become relevant at current times. The fraction $\beta(M)$ can be related to the current density parameters of $\mathrm{PBH}$ and radiation, $\Omega_{\mathrm{PBH}}$ and $\Omega_{\gamma}$, as (Carr et al., 2010),

$$
\Omega_{\mathrm{PBH}}(M)=\beta(M)\left(1+z_{f}\right) \Omega_{\gamma} \simeq \gamma^{1 / 2}\left[\frac{\beta(M)}{1.15 \times 10^{-8}}\right]\left(\frac{M}{M_{\odot}}\right)^{-1 / 2} .
$$

For initial fractions as low as $\beta \sim 10^{-8}$ of solar mass BHs, the fraction of energy in $\mathrm{PBH}$ could be, thus, of order unity.
Depending on the specific mechanism of formation, a population of $\mathrm{PBHs}$ with different masses could be generated. The specific shape of the enhancement of fluctuations determines the mass distribution function. Sharp peaks in the power spectrum imply approximately monochromatic distributions. For instance, chaotic new inflation may give rise to relatively narrow peaks (Yokoyama, 1998; Saito et al., 2008). However, inflation models with an inflection point in a plateau of the potential (GarcíaBellido, 2017; García-Bellido and Ruiz Morales, 2017), or hybrid inflation (Clesse and García-Bellido, 2015), predict, instead, extended mass functions, which can span over a large range of PBHs masses. In this review, we focus on monochromatic distributions for simplicity, although it is possible to translate constraints to extended mass functions, which can be very stringent despite the fact of having more parameters to fit (Carr et al., 2017; Bellomo et al., 2018). Nonetheless, even if there are bounds that exclude $f_{\mathrm{PBH}} \sim 1$ in the monochromatic case, there are choices for the mass function which allow $\mathrm{PBH}$ to constitute most or all of the DM abundance (Lehmann et al., 2018; Ashoorioon et al., 2020; Sureda et al., 2020).

\section{ACCRETION ONTO PBHS}

One of the consequences of the existence of PBHs with greater impact on different observables is the process of accretion. Infalling matter onto $\mathrm{PBHs}$ would release radiation, injecting energy into the surrounding medium, and strongly impacting its thermal state, leaving significant observable signatures. The physics of accretion is highly complex, but one can attempt a simplified approach considering the spherical non-relativistic limit, following the seminal work by Bondi (1952). In this framework, the $\mathrm{BH}$ is treated as a point mass surrounded by matter, embedded in a medium which tends to constant density far enough from the $\mathrm{BH}$. The relevant scale is the so-called Bondi radius, defined by

$$
r_{\mathrm{B}}=\frac{G M_{\mathrm{PBH}}}{c_{s, \infty}^{2}},
$$

where $c_{s, \infty}$ is the speed of sound far enough from the PBH. At distances around $\sim r_{\mathrm{B}}$, the accretion process starts to become 
relevant, and the velocity of the accreted matter reaches $v \sim c_{s, \infty}$, when the density is still close to the boundary value, $\rho_{\infty}$. Taking into account the velocity of the $\mathrm{BH}$ relative to the medium, $v_{\text {rel }}$, one can write the accretion rate as (Bondi, 1952)

$$
\dot{M}_{\mathrm{PBH}}=4 \pi \lambda \rho_{\infty} \frac{\left(G M_{\mathrm{PBH}}\right)^{2}}{\left(c_{s, \infty}^{2}+v_{\mathrm{rel}}^{2}\right)^{3 / 2}},
$$

where the parameter $\lambda$ is the dimensionless accretion rate and depends on the equation of state, but it is order $\sim 1$ for the cases of interest (Ali-Haïmoud and Kamionkowski, 2017). In the early Universe, $v_{\text {rel }}$ can be estimated as the baryon-DM relative velocity, computed in linear theory. Its root-mean-square value is approximately constant before recombination, dropping linearly with $1+z$ at later times (Ali-Haïmoud and Kamionkowski, 2017).

On the other hand, real BHs spin and form an accreting disk. Thus, the spherical symmetric case may not be applicable. Even though $\mathrm{PBH}$ spins are expected to be small, an accretion disk would form if the angular momentum is large enough to keep matter orbiting at Keplerian orbits at distances much larger than the innermost stable orbits, which are roughy given by the Schwarzschild radius (Agol and Kamionkowski, 2002). Applying this criterion, the formation of accretion disks around $\mathrm{PBH}$ has been suggested to occur if the condition $f_{\mathrm{PBH}} M_{\mathrm{PBH}} / M_{\odot} \ll[(1+z) / 730]^{3}$ is fulfilled (Poulin et al., $2017 b)$. This is satisified for $M_{\mathrm{PBH}} \gtrsim M_{\odot}$ and large enough abundances at the epoch of CMB decoupling or at later times. Some of the results outlined above are still valid if the dimensionless accretion rate $\lambda$ is suppresed by roughly two orders of magnitude, after accounting for viscosity effects and matter outflows through jets (Poulin et al., 2017b).

The matter falling onto $\mathrm{BHs}$ is greatly accelerated, which gives rise to radiative emission of high-energy photons by processes such as bremsstrahlung. The luminosity of accreting $\mathrm{BHs}$ is proportional to its accretion rate, and can be written as (Xie and Yuan, 2012)

$$
L_{\mathrm{acc}}=\epsilon\left(\dot{M}_{\mathrm{PBH}}\right) \dot{M}_{\mathrm{PBH}}
$$

where $\epsilon\left(\dot{M}_{\mathrm{PBH}}\right)$ denotes the radiative efficiency, which is in general a complicated function of the accretion rate $\dot{M}_{\mathrm{PBH}}$, and depends upon the geometry, viscosity and other hydrodynamical considerations.

If the accretion disk is optically thin, most of the energy released through viscous dissipation is radiated away, and the luminosities obtained can be close to the Eddington luminosity, $L_{\text {Edd }}$, explaining the extreme brightness of many far Active Galactic Nuclei (AGN) (Shakura and Sunyaev, 1973). However, $\mathrm{PBHs}$, like nearby astrophysical $\mathrm{BHs}$, may radiate in a much less efficient way, through the Advective-DominatedAccretion-Flow (ADAF) (Ichimaru, 1977; Rees et al., 1982; Narayan and Yi, 1994; Narayan and Yi, 1995) (see, e.g., Yuan and Narayan, 2014 for a review). In this scenario, the dynamics is ruled by advective currents, forming a hot thick disk. Most of the emitted energy is deposited in the same accretion disk, heating it up. Thus, only a small portion of energy is released to the surrounding medium, the radiative process being inefficient. In the ADAF scenario, the efficiency function can be fitted by a broken power-law formula, with the slopes and amplitudes dependent on the mass range and the specific modeling of viscosity effects (Xie and Yuan, 2012).

Finally, the energy emitted in the accretion processes is deposited through different channels into the medium. The energy deposition rate for each channel reads (Ali-Haïmoud and Kamionkowski, 2017; Poulin et al., 2017b)

$$
\left(\frac{d E_{c}}{d V d t}\right)_{\mathrm{dep}}=f_{c}(z) L_{\mathrm{acc}} n_{\mathrm{PBH}}=f_{c}(z) L_{\mathrm{acc}} \frac{f_{\mathrm{PBH}} \rho_{\mathrm{DM}}}{M_{\mathrm{PBH}}},
$$

where the subscript $c$ denotes the channel in which energy is deposited, namely: ionization, heating, or atomic excitations (where the Ly $\alpha$ transitions are the most relevant ones). The energy deposition factors $f_{c}(z)$ quantify the fraction of energy which goes to the different channels, and has been computed numerically (Slatyer, 2016). This energy injection would affect different types of observables, as we briefly outline below.

\section{OTHER GENERIC FEATURES}

A noteworthy phenomenon of $\mathrm{BHs}$ is that of evaporation. Due to quantum effects in curved spacetimes, BHs may emit particles at their event horizon, as was noticed in Hawking (1974). The emitted radiation would have a nearly thermal black body spectrum, with a temperature given by (Carr et al., 2010)

$$
T_{\mathrm{BH}}=\frac{\hbar c^{3}}{8 \pi k_{B} G M} \sim 10^{-7} \mathrm{~K} \frac{M_{\odot}}{M},
$$

which is known as Hawking temperature. Due to this emission, BHs would slowly lose mass until completely evaporate. The lifetime of a $\mathrm{PBH}$ of initial mass $M$ is (Lopresto, 2003)

$$
\tau(M) \sim 10^{64} \operatorname{yr}\left(\frac{M}{M_{\odot}}\right)^{3} .
$$

Thus, the lower the PBH mass, the earlier it evaporates. Those with masses of $10^{15} \mathrm{~g}$ or below would have already evaporated by now, having lifetimes shorter than the age of the Universe (Page, 1976), so they cannot contribute to the current DM abundance. These evaporation products or the effects they produce in different observables can be search for in a variety of experiments, probing different mass ranges. Detailed computations of the emitted spectra can be performed by codes such as BlackHawk Arbey and Auffinger (2019).

Another important feature is that of clustering. If fluctuations are originally Gaussian distributed and around a relatively narrow peak, $\mathrm{PBH}$ s are not expected to be originated in clusters, being initially randomly distributed on small scales (Ali-Haïmoud, 2018; Desjacques and Riotto, 2018). However, either primordial non-gaussianities or a broad peak in the power spectrum could lead to a significant initial clustering (Ballesteros et al., 2018; Suyama and Yokoyama, 2019) 
[although broad spectra have also been argued not to produce appreciable clustering (Moradinezhad Dizgah et al., 2019)]. Anyway, PBHs could become bounded as the Universe evolves. A proper determination of their clustering properties at later times is of great importance, for instance, in order to estimate their merger rates (Desjacques and Riotto, 2018; De Luca et al., 2020a). Indeed, the formation of clusters could alleviate some constraints on the PBH abundance (Belotsky et al., 2019).

On another hand, since PBHs would be formed from the collapse of high density peaks relatively spherically symmetric, their torques and angular momentum are expected to be small (Chiba and Yokoyama, 2017; Mirbabayi et al., 2020). It is usually quantified with the dimensionless spin parameter, $\mathcal{S}=S /\left(G M_{\mathrm{PBH}}^{2}\right)$, where $S$ is the spin. Estimations of for PBHs show that it is a small quantity, equal or lower than 0.01 (De Luca et al., 2019a). In contrast, astrophysical BHs are expected to have substantially larger spins, since angular momentum must be conserved during the collapse of their stars of origin, which are often rotating. Hence, the spin can serve as a good proxy to distinguish the nature of a population of BHs. The measurement of low spin parameters could represent a hint for the detection of PBHs. The latest Bayesian analyses of LIGO/Virgo mergers suggest that low values of the spin parameter are strongly preferred by data, regardless of the assumed priors (Garcia-Bellido et al., 2020). Note, however, that the PBH mass and spin depend on the accretion mechanism and their time evolution is correlated (De Luca et al., 2020c).

Furthermore, due to the discrete nature of PBHs, a Poisson shot noise contribution to the matter power spectrum, constant in wavenumber, $\quad P_{\mathrm{sn}}(k) \propto f_{\mathrm{PBH}}^{2} \bar{n}_{\mathrm{PBH}}^{-1} \propto f_{\mathrm{PBH}} M_{\mathrm{PBH}}$, would be expected (Afshordi et al., 2003; Gong and Kitajima, 2017). $\mathrm{PBHs}$ fluctuations give rise to isocurvature modes (Afshordi et al., 2003; Chisholm, 2006; Inman and Ali-Haïmoud, 2019), and thus, affect only at scales smaller than the horizon at the epoch of matter-radiation equality (Peacock, 1999). Therefore, this leads to an enhancement in the matter power spectrum, increasing the population of low-mass halos, which can be constrained by large scale structure and $\operatorname{Ly} \alpha$ forest analyses. This effect is different from the one induced by other nonCDM candidates, such as warm DM or fuzzy DM, which suppress small scale fluctuations, washing out small structures. This contribution becomes relevant for low-mass halos not large enough to cool and collapse to form stars, which are commonly known as minihalos. It has been argued that this enhancement may produce a non-negligible $21 \mathrm{~cm}$ signal from the neutral hydrogen in minihalos (Gong and Kitajima, 2017, Gong and Kitajima, 2018). However, consistently accounting for the heating of the IGM due to PBH accretion increases the Jeans mass and suppresses the minihalo $21 \mathrm{~cm}$ signal, making it almost negligible (Mena et al., 2019).

\section{OBSERVATIONAL CONSTRAINTS ON PBHS AS DM}

PBHs can impact cosmology and astrophysics in a wide range of ways, leaving different observational effects which allow to

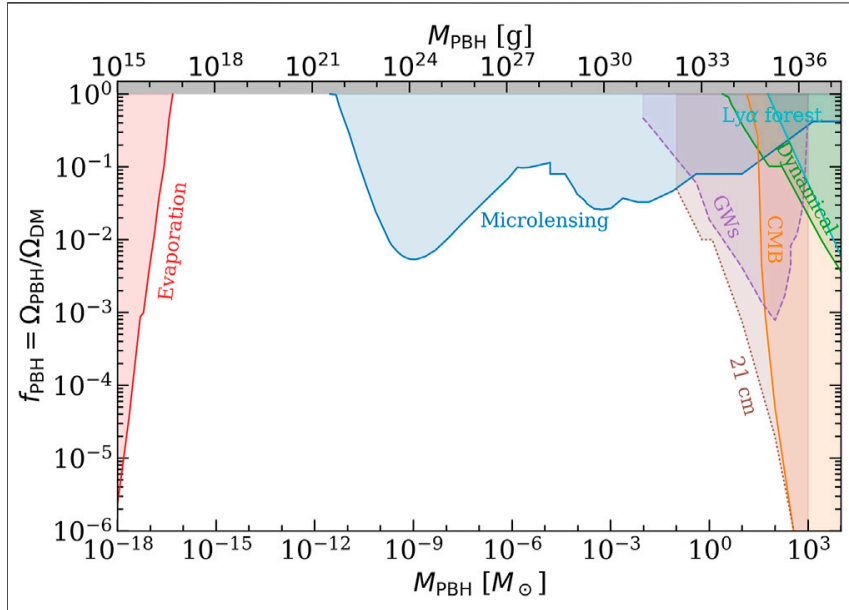

FIGURE 2 | Compilation of constraints on the $\mathrm{PBH}$ fraction (with respect to $\mathrm{DM}$ ) as a function of the $\mathrm{PBH}$ mass, assuming a monochromatic mass function. The different probes considered are: impact of $\mathrm{PBH}$ evaporation (red) on the extragalactic $\gamma$-ray background (Carr et al., 2010) and on the CMB spectrum (Clark et al., 2017); non-observation of microlensing events (blue) from the MACHO (Alcock et al., 2001), EROS (Tisserand et al., 2007), Kepler (Griest et al., 2014), Icarus (Oguri et al., 2018), OGLE (Niikura et al., 2019b) and Subaru-HSC (Croon et al., 2020) collaborations; PBH accretion signatures on the $\mathrm{CMB}$ (orange), assuming spherical accretion of $\mathrm{PBH}$ within halos (Serpico et al., 2020); dynamical constraints, such as disruption of stellar systems by the presence of $\mathrm{PBH}$ (green), on wide binaries (Monroy-

Rodríguez and Allen, 2014) and on ultra-faint dwarf galaxies (Brandt, 2016); power spectrum from the Ly $\alpha$ forest (cyan) (Murgia et al., 2019); merger rates from gravitational waves (purple), either from individual mergers (Kavanagh et al., 2018; Abbott et al., 2019) or from searches of stochastic gravitational wave background (Chen and Huang, 2020). Gravitational waves limits are denoted by dashed lines, since they could be invalidated (Boehm et al., 2021). Dotted brown line corresponds to forecasts from the $21 \mathrm{~cm}$ power spectrum with SKA sensitivities (Mena et al., 2019) and from $21 \mathrm{~cm}$ forest prospects (Villanueva-Domingo and Ichiki, 2021). Figure created with the publicly available Python code PBHbounds (Kavanagh, 2019).

constrain their properties. In this section, we review the most important bounds on the current fraction of $\mathrm{PBH}$ as $\mathrm{DM}$, $f_{\mathrm{PBH}}=\Omega_{\mathrm{PBH}} / \Omega_{\mathrm{DM}}$, for a wide range of masses $M_{\mathrm{PBH}}$, for monochromatic mass functions. A collection of limits from the different probes is depicted in Figure 2. For a more comprehensive list of constraints, see Green and Kavanagh (2021); Carr and Kühnel (2020).

\section{Evaporation}

Since BHs emit energy due to Hawking radiation, those with a lifetime shorter than the age of the Universe must have disintegrated nowadays, a fact which excludes PBHs with $M_{\mathrm{PBH}}<M \simeq 4 \times 10^{14} \mathrm{~g}$ to form part of the current DM (Page, 1976). Moreover, PBHs with masses small enough, although still present, should emit a strong $\gamma$ ray and cosmic ray background which could be observed. Absence of its detection strongly constrains the range of masses $M_{\mathrm{PBH}} \lesssim 10^{17} \mathrm{~g}$. In particular, the maximum fraction allowed is $f_{\mathrm{PBH}} \lesssim 2 \times 10^{-8}\left(M_{\mathrm{PBH}} / M_{*}\right)^{3+\epsilon}$, with $\epsilon \sim 0.1-0.4$ (Carr et al., 2016). Comparable limits have been found from INTEGRAL and COMPTEL observations of the Galactic Center (DeRocco and Graham, 2019; Laha, 2019; 
Coogan et al., 2020; Laha et al., 2020). Furthermore, bounds from the isotropic X-ray and soft gamma-ray background have also been recently updated (Ballesteros et al., 2020; Iguaz et al., 2021). Additionally, data on the diffuse supernova neutrino background at Super-Kamiokande are also able to set constraints (Dasgupta et al., 2020).

\section{Microlensing}

If a compact object crosses the line of sight of a star, it may produce a so-called microlensing effect, which implies a transient and achromatic amplification of its flux. The range of masses of the objects which can produce it span from $5 \times 10^{-10}$ to $\sim 100 M_{\odot}$ (Paczynski, 1986; Green and Kavanagh, 2021). The non-detection of these events leads to bounds on the maximum abundance of $\mathrm{PBHs}$ about $f_{\mathrm{PBH}} \lesssim 0.01-0.1$ by the MACHO (Alcock et al., 2001) and EROS (Tisserand et al., 2007) surveys in the Large and Small Magellanic Clouds, the Subaru Hyper Suprime Cam (HSC) in M31 (Andromeda) (Niikura et al., 2019a) and the Optical Gravitational Lensing Experiment (OGLE) in the Galactic bulge (Niikura et al., 2019b). Nonetheless, the existence of Earth-mass PBHs $\left(M_{\mathrm{PBH}} \sim 10^{-5} M_{\odot}\right)$ with a fraction $f_{\mathrm{PBH}} \sim 0.03$ could explain the observed excess of six microlensing events found in the OGLE data (Mróz et al., 2017), which is consistent with other constraints in this range of PBHs masses (Niikura et al., 2019b). Although this may constitute a hint of their existence, it cannot be regarded as a detection of $\mathrm{PBHs}$, since these microlensing observations could also be explained by free-floating planets. There are some caveats regarding the results of the MACHO collaboration (Hawkins, 2015), since the limits reported are model dependent and could be biased by the assumption of an over-massive halo. Moreover, the results of the MACHO and EROS projects have been found to be statistically incompatible. Therefore, these bounds are not completely reliable, and $\mathrm{PBH}$ s could not be definitely ruled out within this range of masses. Variations of the lensing effect in type Ia Supernovae (Zumalacárregui and Seljak, 2018) and gamma-ray bursts (Barnacka et al., 2012) have also been proposed to constrain the $\mathrm{PBH}$ parameter space, although these limits have been later invalidated (Garcia-Bellido et al., 2017; Katz et al., 2018).

\section{Gravitational Waves}

The observation of $\mathrm{BH}$ mergers by LIGO and Virgo collaborations can be employed to constrain the allowed number of PBHs. Demanding that the predicted merger rates of $\mathrm{PBH}$ binaries cannot exceed the ones measured by gravitational waves, tight upper bounds of $f_{\mathrm{PBH}} \lesssim 0.01$ have been found for $\mathrm{PBHs}$ masses between 1 and $300 M_{\odot}$ (AliHaïmoud et al., 2017; Kavanagh et al., 2018). The nonobservation of a stochastic gravitational wave background of mergers expected from a population of $\mathrm{PBHs}$ has also been used to constrain their abundace (Chen and Huang, 2020). However, to derive these limits, $\mathrm{BHs}$ have been treated as Schwarzschild BHs, while it would be more appropiate to use cosmological BH solutions embedded in a FLRW metric, such as the Thakurta metric (Thakurta, 1981). This implies a timedependent mass, and that $\mathrm{PBH}$ binaries created before galaxy formation would have merged at much earlier times, allowing to obtain merger rates consistent with the LIGO observations, and completely avoiding these constraints (Boehm et al., 2021).

\section{Dynamical Constraints}

Due to two-body interactions, kinetic energies of systems of different masses usually become balanced and match. If a stellar system also has an additional MACHO population, its stars would gain kinetic energy and, due to the virial theorem, the system would expand. Therefore, the presence of PBHs would dynamically heat star clusters, making them larger and with higher velocity dispersions, leading to an eventual dissolution into its host galaxy. Populations with high mass to luminosity ratios are more sensitive to this effect, as happens with ultra faint dwarf galaxies (UFDW), which would be disrupted by the presence of PBHs. Making use of these effects, tight bounds have been obtained at $f_{\mathrm{PBH}} \sim 10^{-3}$ for $M_{\mathrm{PBH}} \sim 10^{4} M_{\odot}$, weakening at lower masses down to $f_{\mathrm{PBH}} \lesssim 1$ for $M_{\mathrm{PBH}} \sim 10 M_{\odot}$ (Brandt, 2016). In a similar way, wide binary stellar systems may be perturbed by compact objects, potentially being disrupted after multiple encounters. The separation distribution of wide binaries restricts the $\mathrm{PBH}$ fraction from $f_{\mathrm{PBH}} \lesssim 1$ for $M_{\mathrm{PBH}} \simeq 3 M_{\odot}$ down to $f_{\mathrm{PBH}} \lesssim 0.1$ at $M_{\mathrm{PBH}} \gtrsim 70 M_{\odot}$ (Monroy-Rodríguez and Allen, 2014).

\section{CMB}

Radiation emitted either by accretion or from Hawking evaporation may affect the $\mathrm{CMB}$ spectrum in two ways: producing spectral distortions and modifying temperature anisotropies. The energetic radiation can enhance the ionization rate, delay recombination and shift the peaks of the $\mathrm{CMB}$ anisotropy spectrum, as well as induce more diffusion damping. The polarization spectrum could also be modified, since the increase of the free electron fraction would increase the Thomson optical depth and enhance the reionization bump at large angular scales. Although early CMB analyses (Ricotti et al., 2008) found very stringent bounds on the allowed abundance of accreting $\mathrm{PBHs}$, later works revisited these computations and found much milder constraints (Horowitz, 2016; Ali-Haïmoud and Kamionkowski, 2017). On another hand, while the former constraints rely on the assumption of spherical accretion, accreting disks have been argued to be more realistic for PBHs, resulting in tighter limits (Poulin et al., 2017b). Taking into account that $\mathrm{PBH}$ could be immersed in DM halos with higher densities than the background, their accretion rates would be increased, also leading to more stringent constraints (Serpico et al., 2020). CMB limits from accretion are currently the most stringent ones for masses $\gtrsim 10 M_{\odot}$. The main caveat is their dependence on some details of the accretion mechanisms, such as the effective velocity and the accretion rate, which may not be very well understood yet.

On the other hand, the energy injection from $\mathrm{PBH}$ evaporation would produce anisotropies and spectral distortions in the $\mathrm{CMB}$ spectrum, which would also limit the maximum abundance, leading to similar costraints to those obtained from the extra-galactic $\gamma$-ray background commented above (Clark et al., 2017; Poulin et al., 2017a; Acharya and Khatri, 2020). Besides energy injection from accretion of $\mathrm{BH}$ evaporation, 
spectral distortions can also be produced by other means, such as the diffusion of photons due to Silk damping at small scales. This allows the translation of constraints on spectral distortions from FIRAS to stringent upper bounds on the $\mathrm{PBH}$ abundance, for masses $M_{\mathrm{PBH}}>10^{5} M_{\odot}$ (Nakama et al., 2018).

\section{Ly $\alpha$ Forest}

The discrete nature of $\mathrm{PBH}$ sould lead to a shot-noize contribution to the matter power spectrum, enchancing small scale fluctuations. Observations of the Ly $\alpha$ forest, which traces matter distribution at small scales, have been employed to extract limits on the maximum allowed fraction of $\mathrm{PBHs}$ (Afshordi et al., 2003). The shot-noize contribution to the power spectrum depends on the joint product $f_{\mathrm{PBH}} M_{\mathrm{PBH}}$, for which the upper bound $f_{\mathrm{PBH}} M_{\mathrm{PBH}} \leq 60 M_{\odot}$ has been obtained (Murgia et al., 2019). The drawback of this method is on the priors of the reionization modeling and, as any $\operatorname{Ly} \alpha$ forest analysis, is model dependent.

\section{$21 \mathrm{~cm}$ Cosmology}

The $21 \mathrm{~cm}$ line signal from the hyperfine structure of the hydrogen is highly sensitive to the thermal state of the IGM, and thus, energy injection from $\mathrm{PBH}$ accretion or evaporation may leave strong observable signatures. The first claimed measurement of a global absorption dip by the EDGES collaboration (Bowman et al., 2018) may lead to competitive bounds on the $\mathrm{PBH}$ abundance, either from accretion processes (Hektor et al., 2018) or from energy injection by evaporation (Clark et al., 2018; Halder and Banerjee, 2021; Halder and Pandey, 2021). It must be noted, however, that the EDGES signal has not been confirmed yet by other experiments, and it has been argued that it could be explained by alternative mechanisms (Hills et al., 2018; Bradley et al., 2019; Sims and Pober, 2020). On the other hand, although the cosmological $21 \mathrm{~cm}$ power spectrum has not been detected yet, forecasts with future experiments such as HERA and SKA have shown that $21 \mathrm{~cm}$ power spectrum data from these two radiotelescopes could potentially improve the bounds up to $f_{\mathrm{PBH}}<10^{-2}-10^{-6}$ for masses above $M_{\odot}$ (Mena et al., 2019). The $21 \mathrm{~cm}$ forest observed as absorption troughs in the spectra of radio sources at $z \sim 10-15$ could also provide similar limits on the $\mathrm{PBH}$

\section{REFERENCES}

Abbott, B. P., Abbott, R., Abbott, T., Abbott, D., Abernathy, M. R., Acernese, K., et al. (2016). Observation of Gravitational Waves from a Binary Black Hole Merger. Phys. Rev. Lett. 116, 061102. doi:10.1103/PhysRevLett.116. 061102

Abbott, B. P., Abbott, R., Abbott, T. D., Abbott, S., Abraham, F., Acernese, K., et al. (2019). Search for Subsolar Mass Ultracompact Binaries in Advanced LIGO's Second Observing Run. Phys. Rev. Lett. 123, 161102. doi:10.1103/PhysRevLett. 123.161102

Abbott, R., Abbott, S., Abraham, F., Acernese, K., Ackley, C., Adams, R. X., et al. (2020). GW190521: a Binary Black Hole Merger with a Total Mass of

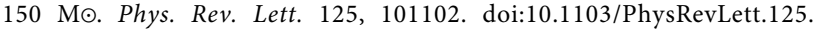
101102

Acharya, S. K., and Khatri, R. (2020). CMB Spectral Distortions Constraints on Primordial Black Holes, Cosmic Strings and Long Lived Unstable Particles Revisited. JCAP 02, 010. doi:10.1088/1475-7516/2020/02/010 abundance, due to the Poisson shot noise and to the accretion heating effect (Villanueva-Domingo and Ichiki, 2021).

\section{CONCLUSION}

The extremely rich physics involved in the formation, evolution and distribution of PBHs implies a large number of observable effects which allow probing them. A myriad of constraints are present for a large range of $\mathrm{PBH}$ masses. In recent years some of these limits, as those from microlensing, femtolensing, CMB accretion or $\mathrm{BH}$ mergers, have been revisited. Some of these bounds have been significantly weakened or have even disappeared, opening windows in the parameter space where $\mathrm{PBH}$ s could still form a substantial part of the DM, if not all. On the other hand, future experiments with better sensitivities may be able to reach yet unexplored regions in the parameter space and tighten up current limits. New probes, such as the $21 \mathrm{~cm}$ line pursued in radio interferometers like SKA, will present a promising and powerful way to proof or refute the existence of $\mathrm{PBH}$ formed in the early universe and their potential contribution to the DM in the Universe.

\section{AUTHOR CONTRIBUTIONS}

PV-D is the main author of the text and the figures. OM and SP-R have substantially collaborated in the editing process.

\section{FUNDING}

PV and OM are supported by the Spanish grants FPA201785985-P and PROMETEO/2019/083. SP is supported by the Spanish FEDER/MCIU-AEI grant FPA2017-84543-P, and partially, by the Portuguese FCT (UID/FIS/00777/2019 and CERN/FIS-PAR/0004/2019). All authors acknowledge support from the European ITN project HIDDeN (H2020-MSCA-ITN2019//860881-HIDDeN).

Afshordi, N., McDonald, P., and Spergel, D. N. (2003). Primordial Black Holes as Dark Matter: the Power Spectrum and Evaporation of Early Structures. ApJ 594, L71-L74. doi:10.1086/378763

Agol, E., and Kamionkowski, M. (2002). X-rays from Isolated Black Holes in the Milky Way. Mon. Not. Roy. Astron. Soc. 334, 553. doi:10.1046/j.1365-8711. 2002.05523.x

Alcock, C., Allsman, R. A., Alves, D. R., Axelrod, T. S., Becker, A. C., Bennett, D. P., et al. (2001). MACHO Project Limits on Black Hole Dark Matter in the 1-30 M Range. Astrophys. J. Lett. 550, L169. doi:10.1086/319636

Ali-Haïmoud, Y. (2018). Correlation Function of High-Threshold Regions and Application to the Initial Small-Scale Clustering of Primordial Black Holes. Phys. Rev. Lett. 121, 081304. doi:10.1103/PhysRevLett.121.081304

Ali-Haïmoud, Y., and Kamionkowski, M. (2017). Cosmic Microwave Background Limits on Accreting Primordial Black Holes. Phys. Rev. D95, 043534. doi:10. 1103/PhysRevD.95.043534

Ali-Haïmoud, Y., Kovetz, E. D., and Kamionkowski, M. (2017). Merger Rate of Primordial Black-Hole Binaries. Phys. Rev. D 96, 123523. doi:10.1103/ PhysRevD.96.123523 
Arbey, A., and Auffinger, J. (2019). BlackHawk: a Public Code for Calculating the Hawking Evaporation Spectra of Any Black Hole Distribution. Eur. Phys. J. C 79, 693. doi:10.1140/epjc/s10052-019-7161-1

Ashoorioon, A., Rostami, A., and Firouzjaee, J. T. (2020). "Charting the Landscape in Our Neighborhood from the PBHs Mass Distribution and GWs,".

Atal, V., and Germani, C. (2019). The Role of Non-gaussianities in Primordial Black Hole Formation. Phys. Dark Univ. 24, 100275. doi:10.1016/j.dark.2019. 100275

Ballesteros, G., Coronado-Blázquez, J., and Gaggero, D. (2020). X-ray and Gammaray Limits on the Primordial Black Hole Abundance from Hawking Radiation. Phys. Lett. B 808, 135624. doi:10.1016/j.physletb.2020.135624

Ballesteros, G., Serpico, P. D., and Taoso, M. (2018). On the Merger Rate of Primordial Black Holes: Effects of Nearest Neighbours Distribution and Clustering. J. Cosmology Astropart. Phys. 2018, 043. doi:10.1088/1475-7516/ 2018/10/043

Barnacka, A., Glicenstein, J. F., and Moderski, R. (2012). New Constraints on Primordial Black Holes Abundance from Femtolensing of Gamma-ray Bursts. Phys. Rev. D 86, 043001. doi:10.1103/PhysRevD.86.043001

Bellomo, N., Bernal, J. L., Raccanelli, A., and Verde, L. (2018). Primordial Black Holes as Dark Matter: Converting Constraints from Monochromatic to Extended Mass Distributions. J. Cosmology Astropart. Phys. 2018, 004. doi:10.1088/1475-7516/2018/01/004

Belotsky, K. M., Dokuchaev, V. I., Eroshenko, Y. N., Esipova, E. A., Khlopov, M. Y., Khromykh, L. A., et al. (2019). Clusters of Primordial Black Holes. Eur. Phys. J. C 79, 246. doi:10.1140/epjc/s10052-019-6741-4

Belotsky, K. M., Dmitriev, A. D., Esipova, E. A., Gani, V. A., Grobov, A. V., et al. (2014). Signatures of Primordial Black Hole Dark Matter. Mod. Phys. Lett. A. 29, 1440005. doi:10.1142/S0217732314400057

Bertone, G., and Hooper, D. (2018). History of Dark Matter. Rev. Mod. Phys. 90, 045002. doi:10.1103/RevModPhys.90.045002

Bird, S., Cholis, I., Muñoz, J. B., Ali-Haïmoud, Y., Kamionkowski, M., et al. (2016). Did LIGO Detect Dark Matter? Phys. Rev. Lett. 116, 201301. doi:10.1103/ PhysRevLett.116.201301

Boehm, C., Kobakhidze, A., O'hare, C. A. J., Picker, Z. S. C., and Sakellariadou, M. (2021). Eliminating the LIGO Bounds on Primordial Black Hole Dark Matter. JCAP 03, 078. doi:10.1088/1475-7516/2021/03/078

Bondi, H. (1952). On Spherically Symmetrical Accretion. MNRAS 112, 195. doi:10. 1093/mnras/112.2.195

Bowman, J. D., Rogers, A. E. E., Monsalve, R. A., Mozdzen, T. J., and Mahesh, N. (2018). An Absorption Profile Centred at 78 Megahertz in the Sky-Averaged Spectrum. Nature 555, 67-70. doi:10.1038/nature25792

Bradley, R. F., Tauscher, K., Rapetti, D., and Burns, J. O. (2019). A Ground Plane Artifact that Induces an Absorption Profile in Averaged Spectra from Global 21 Cm Measurements, with Possible Application to EDGES. ApJ 874, 153. doi:10. 3847/1538-4357/ab0d8b

Brandt, T. D. (2016). Constraints on MACHO Dark Matter from Compact Stellar Systems in Ultra-faint dwarf Galaxies. ApJ 824, L31. doi:10.3847/2041-8205/ $824 / 2 / \mathrm{L} 31$

Cai, R.-g., Pi, S., and Sasaki, M. (2019). Gravitational Waves Induced by Nongaussian Scalar Perturbations. Phys. Rev. Lett. 122, 201101. doi:10.1103/ PhysRevLett.122.201101

Carr, B. J. (1975). The Primordial Black Hole Mass Spectrum. ApJ 201, 1-19. doi: $10.1086 / 153853$

Carr, B. J., Kohri, K., Sendouda, Y., and Yokoyama, J. (2010). New Cosmological Constraints on Primordial Black Holes. Phys. Rev. D 81, 104019. doi:10.1103/ PhysRevD.81.104019

Carr, B., Kohri, K., Sendouda, Y., and Yokoyama, J. (2020). "Constraints on Primordial Black Holes,".

Carr, B., and Kühnel, F. (2020). Primordial Black Holes as Dark Matter: Recent Developments. Annu. Rev. Nucl. Part. Sci. 70, 355-394. doi:10.1146/annurevnucl-050520-125911

Carr, B., Kühnel, F., and Sandstad, M. (2016). Primordial Black Holes as Dark Matter. Phys. Rev. D 94, 083504. doi:10.1103/PhysRevD.94.083504

Carr, B., Raidal, M., Tenkanen, T., Vaskonen, V., and Veermäe, H. (2017). Primordial Black Hole Constraints for Extended Mass Functions. Phys. Rev. D 96, 023514. doi:10.1103/PhysRevD.96.023514

Carr, B., and Silk, J. (2018). Primordial Black Holes as Generators of Cosmic Structures. MNRAS 478, 3756-3775. doi:10.1093/mnras/sty1204
Chapline, G. F. (1975). Cosmological Effects of Primordial Black Holes. Nat. J. C 253, 251-252. doi:10.1038/253251a0

Chen, Z.-C., and Huang, Q.-G. (2020). Distinguishing Primordial Black Holes from Astrophysical Black Holes by Einstein Telescope and Cosmic Explorer. J. Cosmology Astropart. Phys. 2020, 039. doi:10.1088/1475-7516/2020/08/039

Chiba, T., and Yokoyama, S. (2017). Spin Distribution of Primordial Black Holes. PTEP 2017, 083E01. doi:10.1093/ptep/ptx087

Chisholm, J. R. (2006). Clustering of Primordial Black Holes: Basic Results. Phys. Rev. D 73, 083504. doi:10.1103/PhysRevD.73.083504

Clark, S., Dutta, B., Gao, Y., Ma, Y.-Z., and Strigari, L. E. (2018). 21 Cm Limits on Decaying Dark Matter and Primordial Black Holes. Phys. Rev. D 98, 043006. doi:10.1103/PhysRevD.98.043006

Clark, S., Dutta, B., Gao, Y., Strigari, L. E., and Watson, S. (2017). Planck Constraint on Relic Primordial Black Holes. Phys. Rev. D 95, 083006. doi:10.1103/ PhysRevD.95.083006

Clesse, S., and García-Bellido, J. (2015). Massive Primordial Black Holes from Hybrid Inflation as Dark Matter and the Seeds of Galaxies. Phys. Rev. D 92, 023524. doi:10.1103/PhysRevD.92.023524

Clesse, S., and García-Bellido, J. (2018). Seven Hints for Primordial Black Hole Dark Matter. Phys. Dark Universe 22, 137-146. doi:10.1016/j.dark.2018.08.004

Clesse, S., and García-Bellido, J. (2017). The Clustering of Massive Primordial Black Holes as Dark Matter: Measuring Their Mass Distribution with Advanced LIGO. Phys. Dark Univ. 15, 142-147. doi:10.1016/j.dark.2016.10.002

Coogan, A., Morrison, L., and Profumo, S. (2020). Direct Detection of Hawking Radiation from Asteroid-Mass Primordial Black Holes. Phys. Rev. Lett. 126, 171101. doi:10.1103/PhysRevLett.126.171101

Croon, D., McKeen, D., Raj, N., and Wang, Z. (2020). Subaru-HSC through a Different Lens: Microlensing by Extended Dark Matter Structures. Phys. Rev. D 102, 083021. doi:10.1103/PhysRevD.102.083021

Dasgupta, B., Laha, R., and Ray, A. (2020). Neutrino and Positron Constraints on Spinning Primordial Black Hole Dark Matter. Phys. Rev. Lett. 125, 101101. doi:10.1103/PhysRevLett.125.101101

De Luca, V., Desjacques, V., Franciolini, G., Malhotra, A., and Riotto, A. (2019a). The Initial Spin Probability Distribution of Primordial Black Holes. J. Cosmology Astropart. Phys. 2019, 018. doi:10.1088/1475-7516/2019/05/018

De Luca, V., Desjacques, V., Franciolini, G., and Riotto, A. (2020a). The Clustering Evolution of Primordial Black Holes. JCAP 11, 028. doi:10.1088/1475-7516/ 2020/11/028

De Luca, V., Franciolini, G., Kehagias, A., Peloso, M., Riotto, A., and Ünal, C. (2019b). The Ineludible Non-gaussianity of the Primordial Black Hole Abundance. J. Cosmology Astropart. Phys. 2019, 048. doi:10.1088/1475-7516/2019/07/048

De Luca, V., Franciolini, G., Pani, P., and Riotto, A. (2021). Bayesian Evidence for Both Astrophysical and Primordial Black Holes: Mapping the GWTC-2 Catalog to Third-Generation Detectors, J. Cosmology Astroparticle Phys., 2021, 003. doi:10.1088/1475-7516/2021/05/003

De Luca, V., Franciolini, G., Pani, P., and Riotto, A. (2020b). Primordial Black Holes Confront LIGO/Virgo Data: Current Situation. JCAP 06, 044. doi:10. 1088/1475-7516/2020/06/044

De Luca, V., Franciolini, G., Pani, P., and Riotto, A. (2020c). The Evolution of Primordial Black Holes and Their Final Observable Spins. JCAP 04, 052. doi:10. 1088/1475-7516/2020/04/052

Deng, H., Garriga, J., and Vilenkin, A. (2017). Primordial Black Hole and Wormhole Formation by Domain walls. J. Cosmology Astropart. Phys. 2017, 050. doi:10.1088/1475-7516/2017/04/050

Deng, H., and Vilenkin, A. (2017). Primordial Black Hole Formation by Vacuum Bubbles. J. Cosmology Astropart. Phys. 2017, 044. doi:10.1088/1475-7516/2017/ $12 / 044$

DeRocco, W., and Graham, P. W. (2019). Constraining Primordial Black Hole Abundance with the Galactic $511 \mathrm{keV}$ Line. Phys. Rev. Lett. 123, 251102. doi:10. 1103/PhysRevLett.123.251102

Desjacques, V., and Riotto, A. (2018). Spatial Clustering of Primordial Black Holes. Phys. Rev. D 98, 123533. doi:10.1103/PhysRevD.98.123533

Dolgov, A. D., Kuranov, A. G., Mitichkin, N. A., Porey, S., Postnov, K. A., Sazhina, O. S., et al. (2020). On Mass Distribution of Coalescing Black Holes. JCAP 12, 017. doi:10.1088/1475-7516/2020/12/017

Escrivà, A., Germani, C., and Sheth, R. K. (2021). Analytical Thresholds for Black Hole Formation in General Cosmological Backgrounds. JCAP 01, 030. doi:10. $1088 / 1475-7516 / 2021 / 01 / 030$ 
Franciolini, G., Kehagias, A., Matarrese, S., and Riotto, A. (2018). Primordial Black Holes from Inflation and Non-gaussianity. J. Cosmology Astropart. Phys. 2018. 016 doi:10.1088/1475-7516/2018/03/016

García-Bellido, J. (2017). Massive Primordial Black Holes as Dark Matter and Their Detection with Gravitational Waves. J. Phys. Conf. Ser. 840, 012032. doi:10. 1088/1742-6596/840/1/012032

Garcia-Bellido, J., Clesse, S., and Fleury, P. (2017). LIGO Lo(g)Normal MACHO: Primordial Black Holes Survive SN Lensing Constraints. Phys. Dark Univ. 20, 95-100. doi:10.1016/j.dark.2018.04.005

Garcia-Bellido, J., Nuño Siles, J. F., and Ruiz Morales, E. (2020). Bayesian Analysis of the Spin Distribution of LIGO/Virgo Black Holes. Phys. Dark Universe 31, 100791. doi:10.1016/j.dark.2021.100791

García-Bellido, J., and Ruiz Morales, E. (2017). Primordial Black Holes from Single Field Models of Inflation. Phys. Dark Universe 18, 47-54. doi:10.1016/j.dark. 2017.09.007

Garriga, J., Vilenkin, A., and Zhang, J. (2016). Black Holes and the Multiverse. J. Cosmology Astropart. Phys. 2016, 064. doi:10.1088/1475-7516/2016/02/064

Germani, C., and Musco, I. (2019). Abundance of Primordial Black Holes Depends on the Shape of the Inflationary Power Spectrum. Phys. Rev. Lett. 122, 141302. doi:10.1103/PhysRevLett.122.141302

Germani, C., and Sheth, R. K. (2020). Nonlinear Statistics of Primordial Black Holes from Gaussian Curvature Perturbations. Phys. Rev. D 101, 063520. doi:10.1103/PhysRevD.101.063520

Gong, J.-O., and Kitajima, N. (2018). Distribution of Primordial Black Holes and 21cm Signature. JCAP 1811, 041. doi:10.1088/1475-7516/2018/11/041

Gong, J.-O., and Kitajima, N. (2017). Small-scale Structure and $21 \mathrm{~cm}$ Fluctuations by Primordial Black Holes. JCAP 1708, 017. doi:10.1088/1475-7516/2017/ $08 / 017$

Green, A. M., and Kavanagh, B. J. (2021). Primordial Black Holes as a Dark Matter Candidate. J. Phys. G 48, 4. doi:10.1088/1361-6471/abc534

Green, A. M., Liddle, A. R., Malik, K. A., and Sasaki, M. (2004). A New Calculation of the Mass Fraction of Primordial Black Holes. Phys. Rev. D 70, 041502. doi:10. 1103/PhysRevD.70.041502

Green, A. M. (2014). Primordial Black Holes: Sirens of the Early Universe. Quan. Aspects Black Holes 178, 129-149. doi:10.1007/978-3-319-10852-0_5

Griest, K., Cieplak, A. M., and Lehner, M. J. (2014). Experimental Limits on Primordial Black Hole Dark Matter from the First 2 Yr of Kepler Data. Astrophys. J. 786, 158. doi:10.1088/0004-637X/786/2/158

Halder, A., and Banerjee, S. (2021). Bounds on Abundance of Primordial Black Hole and Dark Matter from EDGES 21-cm Signal. Phys. Rev. D 103, 063044. doi:10.1103/PhysRevD.103.063044

Halder, A., and Pandey, M. (2021). Investigating the Effect of PBH, Dark Matter Baryon and Dark Matter - Dark Energy Interaction on EDGES in $21 \mathrm{~cm}$ Signal

Hall, A., Gow, A. D., and Byrnes, C. T. (2020). Bayesian Analysis of LIGO-Virgo Mergers: Primordial vs. Astrophysical Black Hole Populations. Phys. Rev. D 102, 123524. doi:10.1103/PhysRevD.102.123524

Hawking, S. (1989). Black Holes from Cosmic Strings. Phys. Lett. B 231, 237-239. doi:10.1016/0370-2693(89)90206-2

Hawking, S. (1971). Gravitationally Collapsed Objects of Very Low Mass. MNRAS 152, 75. doi:10.1093/mnras/152.1.75

Hawking, S. W. (1974). Black Hole Explosions? Nat. J. C 248, 30-31. doi:10.1038/ 248030a0

Hawking, S. W., Moss, I. G., and Stewart, J. M. (1982). Bubble Collisions in the Very Early Universe. Phys. Rev. D 26, 2681-2693. doi:10.1103/PhysRevD.26.2681

Hawkins, M. R. S. (2015). A New Look at Microlensing Limits on Dark Matter in the Galactic Halo. A\&A 575, A107. doi:10.1051/0004-6361/201425400

Hektor, A., Hütsi, G., Marzola, L., Raidal, M., Vaskonen, V., and Veermäe, H. (2018). Constraining Primordial Black Holes with the EDGES $21-\mathrm{cm}$ Absorption Signal. Phys. Rev. D 98, 023503. doi:10.1103/PhysRevD.98.023503

Hills, R., Kulkarni, G., Meerburg, P. D., and Puchwein, E. (2018). Concerns about Modelling of the EDGES Data. Nat. J. C 564, E32-E34. doi:10.1038/s41586-0180796-5

Horowitz, B. (2016). Revisiting Primordial Black Holes Constraints from Ionization History

Hütsi, G., Raidal, M., Vaskonen, V., and Veermäe, H. (2021). Two Populations of LIGO-Virgo Black Holes. JCAP 03, 068. doi:10.1088/1475-7516/2021/03/068

Ichimaru, S. (1977). Bimodal Behavior of Accretion Disks - Theory and Application to Cygnus X-1 Transitions. Astrophys. J. 214, 840-855. doi:10.1086/155314
Iguaz, J., Serpico, P. D., and Siegert, T. (2021). Isotropic X-ray Bound on Primordial Black Hole Dark Matter

Inman, D., and Ali-Haïmoud, Y. (2019). Early Structure Formation in Primordial Black Hole Cosmologies. Phys. Rev. D 100, 083528. doi:10.1103/PhysRevD.100. 083528

Katz, A., Kopp, J., Sibiryakov, S., and Xue, W. (2018). Femtolensing by Dark Matter Revisited. J. Cosmology Astropart. Phys. 2018, 005. doi:10.1088/1475-7516/ 2018/12/005

Kavanagh, B. J. [Dataset](2019). Bradkav/Pbhbounds: Release version. doi:10. 5281/zenodo.3538999

Kavanagh, B. J., Gaggero, D., and Bertone, G. (2018). Merger Rate of a Subdominant Population of Primordial Black Holes. Phys. Rev. D 98, 023536. doi:10.1103/PhysRevD.98.023536

Ketov, S. V., and Khlopov, M. Y. (2019). Cosmological Probes of Supersymmetric Field Theory Models at Superhigh Energy Scales. Symmetry 11, 511. doi:10. 3390/sym11040511

Khlopov, M. Y. (2010). Primordial Black Holes. Res. Astron. Astrophys. 10, 495-528. doi:10.1088/1674-4527/10/6/001

Laha, R., Muñoz, J. B., and Slatyer, T. R. (2020). INTEGRAL Constraints on Primordial Black Holes and Particle Dark Matter. Phys. Rev. D 101, 123514. doi:10.1103/PhysRevD.101.123514

Laha, R. (2019). Primordial Black Holes as a Dark Matter Candidate Are Severely Constrained by the Galactic center $511 \mathrm{keV} \gamma$-ray Line. Phys. Rev. Lett. 123, 251101. doi:10.1103/PhysRevLett.123.251101

Lehmann, B. V., Profumo, S., and Yant, J. (2018). The Maximal-Density Mass Function for Primordial Black Hole Dark Matter. JCAP 04, 007. doi:10.1088/ $1475-7516 / 2018 / 04 / 007$

Lopresto, M. C. (2003). Some Simple Black Hole Thermodynamics. Phys. Teach. 41, 299-301. doi:10.1119/1.1571268

Mena, O., Palomares-Ruiz, S., Villanueva-Domingo, P., and Witte, S. J. (2019). Constraining the Primordial Black Hole Abundance with 21-cm Cosmology. Phys. Rev. D100, 043540. doi:10.1103/PhysRevD.100.043540

Meszaros, P. (1975). Primeval Black Holes and Galaxy Formation. A $\triangleleft A$ 38, 5-13.

Mirbabayi, M., Gruzinov, A., and Noreña, J. (2020). Spin of Primordial Black Holes. JCAP 03, 017. doi:10.1088/1475-7516/2020/03/017

Monroy-Rodríguez, M. A., and Allen, C. (2014). The End of the MACHO Era Revisited: New Limits on MACHO Masses from Halo Wide Binaries. ApJ 790, 159. doi:10.1088/0004-637X/790/2/159

Moradinezhad Dizgah, A., Franciolini, G., and Riotto, A. (2019). Primordial Black Holes from Broad Spectra: Abundance and Clustering. J. Cosmology Astropart. Phys. 2019, 001. doi:10.1088/1475-7516/2019/11/001

Mróz, P., Udalski, A., Skowron, J., Poleski, R., Kozlowski, S., Szymanski, M. K., et al. (2017). No Large Population of Unbound or Wide-Orbit Jupiter-mass Planets. Nat. J. C 548, 183-186. doi:10.1038/nature23276

Murgia, R., Scelfo, G., Viel, M., and Raccanelli, A. (2019). Lyman- $\alpha$ forest Constraints on Primordial Black Holes as Dark Matter. Phys. Rev. Lett. 123, 071102. doi:10.1103/PhysRevLett.123.071102

Musco, I., De Luca, V., Franciolini, G., and Riotto, A. (2021). Threshold for Primordial Black Holes. II. A Simple Analytic Prescription. Phys. Rev. D 103, 063538. doi:10.1103/PhysRevD.103.063538

Musco, I., Miller, J. C., and Rezzolla, L. (2005). Computations of Primordial Black Hole Formation. Class. Quant. Grav. 22, 1405-1424. doi:10.1088/0264-9381/ 22/7/013

Nakama, T., Carr, B., and Silk, J. (2018). Limits on Primordial Black Holes from $\mu$ Distortions in Cosmic Microwave Background. Phys. Rev. D 97, 043525. doi:10. 1103/PhysRevD.97.043525

Narayan, R., and Yi, I. (1995). Advection Dominated Accretion: Underfed Black Holes and Neutron Stars. Astrophys. J. 452, 710. doi:10.1086/176343

Narayan, R., and Yi, I. (1994). Advection-dominated Accretion: a Self-Similar Solution. ApJ 428, L13. doi:10.1086/187381

Niemeyer, J. C., and Jedamzik, K. (1999). Dynamics of Primordial Black Hole Formation. Phys. Rev. D 59, 124013. doi:10.1103/PhysRevD.59.124013

Niemeyer, J. C., and Jedamzik, K. (1998). Near-critical Gravitational Collapse and the Initial Mass Function of Primordial Black Holes. Phys. Rev. Lett. 80, 5481-5484. doi:10.1103/PhysRevLett.80.5481

Niikura, H., Takada, M., Yasuda, N., Lupton, R. H., Sumi, T., More, S., et al. (2019a). Microlensing Constraints on Primordial Black Holes with Subaru/HSC Andromeda Observations. Nat. Astron. 3, 524-534. doi:10.1038/s41550-019-0723-1 
Niikura, H., Takada, M., Yokoyama, S., Sumi, T., and Masaki, S. (2019b). Constraints on Earth-Mass Primordial Black Holes from OGLE 5-year Microlensing Events. Phys. Rev. D 99, 083503. doi:10.1103/PhysRevD.99.083503

Oguri, M., Diego, J. M., Kaiser, N., Kelly, P. L., and Broadhurst, T. (2018). Understanding Caustic Crossings in Giant Arcs: Characteristic Scales, Event Rates, and Constraints on Compact Dark Matter. Phys. Rev. D 97, 023518. doi:10.1103/PhysRevD.97.023518

Oppenheimer, J. R., and Volkoff, G. M. (1939). On Massive Neutron Cores. Phys. Rev. 55, 374-381. doi:10.1103/PhysRev.55.374

Paczynski, B. (1986). Gravitational Microlensing by the Galactic Halo. ApJ 304, 1. doi:10.1086/164140

Page, D. N. (1976). Particle Emission Rates from a Black Hole. II. Massless Particles from a Rotating Hole. Phys. Rev. D 14, 3260-3273. doi:10.1103/PhysRevD.14. 3260

Peacock, J. A. (1999). Cosmological Physics. Cambridge University Press. doi:10. 1017/CBO9780511804533

Polnarev, A. G., and Khlopov, M. Y. (1985). Cosmology, Primordial Black Holes, and Supermassive Particles. Sov. Phys. Usp. 28, 213-232. doi:10.1070/ PU1985v028n03ABEH003858

Polnarev, A., and Zembowicz, R. (1991). Formation of Primordial Black Holes by Cosmic Strings. Phys. Rev. D 43, 1106-1109. doi:10.1103/PhysRevD.43.1106

Poulin, V., Lesgourgues, J., and Serpico, P. D. (2017a). Cosmological Constraints on Exotic Injection of Electromagnetic Energy. JCAP 03, 043. doi:10.1088/14757516/2017/03/043

Poulin, V., Serpico, P. D., Calore, F., Clesse, S., and Kohri, K. (2017b). CMB Bounds on Disk-Accreting Massive Primordial Black Holes. Phys. Rev. D96, 083524. doi:10.1103/PhysRevD.96.083524

Rees, M. J., Phinney, E. S., Begelman, M. C., and Blandford, R. D. (1982). Ion Supported Tori and the Origin of Radio Jets. Nature 295, 17-21. doi:10.1038/ 295017a0

Ricotti, M., Ostriker, J. P., and Mack, K. J. (2008). Effect of Primordial Black Holes on the Cosmic Microwave Background and Cosmological Parameter Estimates. ApJ 680, 829-845. doi:10.1086/587831

Saito, R., Yokoyama, J., and Nagata, R. (2008). Single-field Inflation, Anomalous Enhancement of Superhorizon Fluctuations and Non-gaussianity in Primordial Black Hole Formation. J. Cosmology Astropart. Phys. 2008, 024. doi:10.1088/ 1475-7516/2008/06/024

Sanders, R. H. (2010). The Dark Matter Problem: A Historical Perspective. Cambridge University Press.

Sasaki, M., Suyama, T., Tanaka, T., and Yokoyama, S. (2016). Primordial Black Hole Scenario for the Gravitational-Wave Wvent GW150914. Phys. Rev. Lett. 117, 061101. doi:10.1103/PhysRevLett.117.061101

Sasaki, M., Suyama, T., Tanaka, T., and Yokoyama, S. (2018). Primordial Black Holes-Perspectives in Gravitational Wave Astronomy. Class. Quant. Grav. 35, 063001. doi:10.1088/1361-6382/aaa7b4

Serpico, P. D., Poulin, V., Inman, D., and Kohri, K. (2020). Cosmic Microwave Background Bounds on Primordial Black Holes Including Dark Matter Halo Accretion. Phys. Rev. Res. 2, 023204. doi:10.1103/PhysRevResearch.2. 023204

Shakura, N. I., and Sunyaev, R. A. (1973). Black Holes in Binary Systems. Observational Appearance. Astron. Astrophys. 24, 337-355.
Sims, P. H., and Pober, J. C. (2020). Testing for Calibration Systematics in the EDGES Low-Band Data Using Bayesian Model Selection. MNRAS 492, 22-38. doi:10.1093/mnras/stz3388

Slatyer, T. R. (2016). Indirect Dark Matter Signatures in the Cosmic Dark Ages II. Ionization, Heating and Photon Production from Arbitrary Energy Injections. Phys. Rev. D93, 023521. doi:10.1103/PhysRevD.93.023521

Sureda, J., Magaña, J., Araya, I. J., and Padilla, N. D. (2020). Press-Schechter Primordial Black Hole Mass Functions and Their Observational Constraints

Suyama, T., and Yokoyama, S. (2019). Clustering of Primordial Black Holes with Non-gaussian Initial Fluctuations. Prog. Theor. Exp. Phys. 2019, 103E02. doi:10. 1093/ptep/ptz105

Thakurta, S. N. G. (1981). Kerr Metric in an Expanding Universe. Indian J. Phys. 55B, 304-310.

Tisserand, P., Le Guillou, L., Afonso, C., Albert, J. N., Andersen, J., Ansari, E., et al. (2007). Limits on the MACHO Content of the Galactic Halo from the EROS-2 Survey of the Magellanic Clouds. Astron. Astrophys. 469, 387-404. doi:10.1051/ 0004-6361:20066017

Tolman, R. C. (1939). Static Solutions of Einstein's Field Equations for Spheres of Fluid. Phys. Rev. 55, 364-373. doi:10.1103/PhysRev.55.364

Villanueva-Domingo, P., and Ichiki, K. (2021). $21 \mathrm{Cm}$ forest Constraints on Primordial Black Holes (Preprint) Arxiv

Volonteri, M. (2010). Formation of Supermassive Black Holes. Astron. Astrophysics Rev. 18, 279-315. doi:10.1007/s00159-010-0029-x

Wong, K. W. K., Franciolini, G., De Luca, V., Baibhav, V., Berti, E., Pani, P., et al. (2021). Constraining the Primordial Black Hole Scenario with Bayesian Inference and Machine Learning: the GWTC-2 Gravitational Wave Catalog. Phys. Rev. D 103, 023026. doi:10.1103/PhysRevD.103.023026

Xie, F.-G., and Yuan, F. (2012). The Radiative Efficiency of Hot Accretion Flows Mon. Not. Roy. Astron. Soc. 427, 1580. doi:10.1111/j.1365-2966.2012.22030.x

Yokoyama, J. (1998). Chaotic New Inflation and Formation of Primordial Black Holes. Phys. Rev. D 58, 083510. doi:10.1103/PhysRevD.58.083510

Yuan, F., and Narayan, R. (2014). Hot Accretion Flows Around Black Holes. Ann. Rev. Astron. Astrophys. 52, 529-588. doi:10.1146/annurev-astro-082812141003

Zel'dovich, Y. B., and Novikov, I. D. (1967). The Hypothesis of Cores Retarded during Expansion and the Hot Cosmological Model. Soviet Ast 10, 602.

Zumalacárregui, M., and Seljak, U. (2018). Limits on Stellar-Mass Compact Objects as Dark Matter from Gravitational Lensing of Type Ia Supernovae. Phys. Rev. Lett. 121, 141101. doi:10.1103/PhysRevLett.121.141101

Conflict of Interest: The authors declare that the research was conducted in the absence of any commercial or financial relationships that could be construed as a potential conflict of interest.

Copyright (C) 2021 Villanueva-Domingo, Mena and Palomares-Ruiz. This is an open-access article distributed under the terms of the Creative Commons Attribution License (CC BY). The use, distribution or reproduction in other forums is permitted, provided the original author(s) and the copyright owner(s) are credited and that the original publication in this journal is cited, in accordance with accepted academic practice. No use, distribution or reproduction is permitted which does not comply with these terms. 